\title{
Harnessing the Potential of Combination Immunotherapy and Oncolytic Virotherapy for Solid Tumors
}

\author{
Sonia Cohen, MD, PhD, and Genevieve M. Boland, MD, PhD $\mathbb{D}$ \\ Department of Surgery, Massachusetts General Hospital, Harvard Medical School, Boston
}

The clinical success of immune checkpoint blockade for melanoma and other solid tumors has accelerated interest in the application of immuno-oncology across cancer types and stages. Oncolytic virotherapy represents a relatively new class of therapeutic agents that exploit the ability of certain viruses to preferentially replicate in and lyse tumor cells, resulting in an inflammatory response in the tumor microenvironment with the potential to induce or accentuate a systemic antitumor response.

Talimogene laherparapvec (T-VEC), a modified herpes virus that expresses granulocyte macrophage colony-stimulating factor (GM-CSF), is the first oncolytic viral therapy in clinical use for cancer in the United States. Approved by the Food and Drug Administration (FDA) in 2015 for use in the local treatment of unresectable, cutaneous, subcutaneous, and nodal melanoma recurrent after initial surgery, treatment with T-VEC is well-tolerated and results in better outcomes than treatment with GM-CSF alone. In particular, for the subgroup of patients with a lower burden of disease at the time of treatment (3B/C or $4 \mathrm{M} 1 \mathrm{a})$, durable response rates of $25.2 \%$ were achieved, with a significant improvement in median survival, from 21.5 to 46.8 months. ${ }^{1}$ However overall survival was not improved for the patients with more advanced metastases and a higher burden of disease. As a result, and with the concurrent introduction of effective systemic therapies for advanced

(C) Society of Surgical Oncology 2021

First Received: 22 October 2021

Accepted: 26 October 2021;

Published Online: 26 November 2021

G. M. Boland, MD, PhD

e-mail: gmboland@mgh.harvard.edu melanoma, the integration of T-VEC into clinical practice has lagged and has been limited to patients with locoregional disease.

The rationale for intratumoral immunotherapy such as oncolytic viruses includes preferential targeting of tumor cells due to viral selectivity, efficient viral replication and tumor cell lysis, induction of tumor-specific antigen presentation leading to innate and adaptive immune stimulation, and induction of a systemic antitumor immune response. This potential for augmenting a widespread antitumor response is the most promising prospect in this era of effective systemic immunotherapies.

Optimal viral oncolysis should generate a polyclonal response to tumor-derived antigens that, in combination with immune checkpoint inhibitors, could enable efficient and durable disease control. Toward this end, efforts are underway to understand how modification of the oncolytic virus itself can augment local tumor lysis, antigen release, and immune recognition. ${ }^{2}$

In addition to herpes simplex virus 1 , a number of other oncolytic viruses are in preclinical and clinical development including adenovirus, reovirus, and poxvirus. Modification of the viral genome to include therapeutic transgenes intended to act in an immune stimulatory manner, similar to GM-CSF, is an additional active avenue of research, as is the modification of the viral genome to enhance tumor specificity and replication efficiency. Finally, a number of ongoing trials are investigating combination therapy with oncolytic virotherapy and systemic immunotherapy to determine whether immune checkpoint inhibition can augment a systemic antitumor response.

Results from the first phase 3 trial addressing the efficacy and safety of combination T-VEC and programmed cell death protein 1 (PD-1) inhibition (Masterkey-265/KEYNOTE-034) were recently reported at the European Society for Medical Oncology (ESMO) Congress 2021 by Dr. Gogas 
and colleagues. The trial randomized 692 patients who had unresectable stage 2B-IVM1c melanoma without prior immunotherapy treatment to T-VEC with pembrolizumab or placebo with pembrolizumab. Addition of T-VEC to pembrolizumab did not significantly improve progression-free or overall survival compared with pembrolizumab treatment alone, suggesting that at least in this context, the addition of $\mathrm{T}$-VEC provided no additional benefit.

These data raise a number of questions regarding the mechanisms whereby oncolytic virus and systemic immunotherapies might interact and how best to harness treatment synergy. Importantly, the results of Masterkey265 show that the clinical context in which combination immunotherapy and oncolytic virotherapy is given matters.

In the retrospective analysis presented in this issue of Annals of Surgical Oncology, Carr and colleagues. address the hypothesis that although T-VEC may not improve outcomes for immunotherapy responders, patients with immunotherapy-resistant advanced melanoma may benefit by the addition of T-VEC. As a rationale, the authors cite evidence that melanomas with primary resistance to immunotherapy have been shown to lack $\mathrm{CD} 8+\mathrm{T}$ cell infiltration on pathologic examination. A T-VEC injection induces melanoma-associated antigen recognized by MART-1-specific CD8 $+\mathrm{T}$ cells. Combining T-VEC with immunotherapy may overcome immunotherapy resistance by stimulating the requisite antigen presentation and $\mathrm{T}$ cell infiltrate in a previously nonresponsive tumor.

Carr and colleagues identified 112 patients with stage 3B-4 melanoma treated with T-VEC after demonstrating progression on immunotherapy, either sequentially or in combination with immunotherapy. The observed disease control rate was $59 \%$, and the median progression-free survival was 9.9 months. Although the authors do not provide a comparison cohort, this is in line with the median progression-free survival reported for T-VEC and pembrolizumab or placebo and pembrolizumab in the context of an immunotherapy-resistant tumor.

A number of ongoing randomized trials should directly address whether the addition of T-VEC results in better outcomes for patients with immunotherapy-resistant advanced melanoma including NCT01740297, NCT02965716, and NCT04068181 (MASTERKEY-115). These data will have particular relevance to other solid tumors with resistance to immunotherapy. If efficacious, priming a "cold" tumor microenvironment with oncolytic virus treatment could revolutionize treatment options in immunotherapy-resistant cancers.

Interestingly the analysis by Carr and colleagues again demonstrated greater efficacy of T-VEC for patients with a lower burden of disease. This repeated observation raises the possibility of using oncolytic viral therapy in resectable disease to stimulate an immune response and prevent disease recurrence by inducing tumor-specific immune surveillance capable of controlling a low burden of disease. Several trials testing the efficacy of neoadjuvant T-VEC either alone or in combination with immunotherapy with respect to recurrence-free survival are ongoing with high-risk primary tumors, locoregional and distant metastases (NCT02211131, NCT04427306, NCT03842943, NCT04330430). These studies will address the hypothesis that the most effective context for combining current oncolytic virotherapy with systemic immunotherapy may be in a neoadjuvant setting. They also will provide molecular correlates for investigating the mechanisms by which oncolytic viral therapy stimulates a systemic antitumor response and allow the design of potentially more effective oncolytic viruses.

Although immune checkpoint blockade has revolutionized the treatment of advanced melanoma, the majority of patients succumb to primary or acquired resistance. Oncolytic viral therapies such as T-VEC provide a promising mechanism for inducing a patient-specific systemic antitumor response that has the potential to overcome the problem of intratumoral heterogeneity. Engineering the viral genome itself to efficiently lyse cancer cells and induce tumor-derived antigen recognition should allow efficient priming of the immune system for an effective and durable systemic response that, in combination with immunotherapy, could provide life-saving treatment not only for immunotherapy-resistant melanomas, but also for a broad range of solid tumors with limited therapeutic options. Toward this end, efforts are ongoing to expand the clinical context and viral payloads to enhance immune activity in historically "cold" tumors.

DISCLOUSURE Genevieve M. Boland has sponsored research agreements with Olink Proteomics, InterVenn Biosciences, Palleon Pharmaceuticals, and Takeda Oncology. She has been a consultant for Merck, Ankyra Therapeutics, and InterVenn Biosciences. She has been on scientific advisory boards for Merck, Novartis, Iovance, Nektar Therapeutics. She was on the steering committee for Nektar Therapeutics.

\section{REFERENCES}

1. Andtbacka RHI, Collichio F, Harrington KJ, Middleton MR, Downey G, Öhrling K, Kaufman HL. Final analyses of OPTiM: a randomized phase III trial of talimogene laherparepvec versus granulocyte-macrophage colony-stimulating factor in unresectable stage III-IV melanoma. $J$ Immunother Cancer. 2019;7:145

2. Macedo N, Miller DM, Haq R, Kaufman HL. Clinical landscape of oncolytic virus research in 2020. J Immunother Cancer. 2020. https://doi.org/10.1136/jitc-2020-001486.

Publisher's Note Springer Nature remains neutral with regard to jurisdictional claims in published maps and institutional affiliations. 